\title{
Expanding Regional Airport Usage to Accommodate Increased Air Traffic Demand
}

\author{
Carl R. Russell ${ }^{*}$ \\ NASA Ames Research Center, Moffett Field, CA, 94035
}

\begin{abstract}
Small regional airports present an underutilized source of capacity in the national air transportation system. This study sought to determine whether a 50 percent increase in national operations could be achieved by limiting demand growth at large hub airports and instead growing traffic levels at the surrounding regional airports. This demand scenario for future air traffic in the United States was generated and used as input to a 24-hour simulation of the national airspace system. Results of the demand generation process and metrics predicting the simulation results are presented, in addition to the actual simulation results. The demand generation process showed that sufficient runway capacity exists at regional airports to offload a significant portion of traffic from hub airports. Predictive metrics forecast a large reduction of delays at most major airports when demand is shifted. The simulation results then show that offloading hub traffic can significantly reduce nationwide delays.
\end{abstract}

\section{Introduction}

$\mathrm{T}$ HE major airports in the United States are beginning to reach or exceed their maximum capacity as overall air traffic levels continue to grow. The top 34 airports in the country, which represent nearly three quarters of all yearly passenger enplanements, are currently predicted to experience a 75 percent increase in demand by the year 2025. The Federal Aviation Administration has put plans in place for specific improvements at some of these airports, but these changes will not generate sufficient capacity to accommodate the increased demand. ${ }^{2}$

Due to physical, environmental, economic, and political constraints, large hub airports are limited in the amount they can expand their operations. There exist, however, several thousand regional airports scattered throughout the national air transportation system. These currently handle only a fraction of their existing runway capacities. Operations at these airports are typically unscheduled general aviation flights.

A possible future scenario involves the more widespread use of these smaller airports to accept increased demand in metropolitan areas. The main underlying assumption in this scenario is that airlines will be willing to extend service beyond their traditional hub and spoke routes. A demand shift to these regional airports is beginning to happen in the current system. Airports such as Stewart Airport in Newburgh, NY are already serving large metropolitan areas where the main hubs are reaching their capacity limits. ${ }^{3}$

Previous studies have looked at several examples to investigate the process by which a regional airport begins to handle increased demand in a metropolitan area. ${ }^{4,5}$ This emergence phenomenon can take several years, but it generally begins with a single airline starting service at an airport and others following suit - a process commonly called the "Southwest Effect." Another previous study focused on developing a suite of seven concepts to accommodate a $2 \mathrm{x}$ increase in air traffic across the system. ${ }^{6}$ While this work included the extended use of regional airports as a capacity enabler, it did not isolate the benefit of shifting demand from hubs to regional airports.

The goal of this study was to assess the system-wide capacity increase enabled by offloading excess demand growth to regional airports if only the FAA's planned improvements are implemented at hub airports. Software was used to grow traffic and offload flights to generate an input demand set. A simulation of the national air transportation system was then used to represent all itinerant traffic for an entire day at current and future demand levels. For comparison, future traffic was simulated with and without flight offloading. Simulation outputs were analyzed to evaluate the performance of the system under both demand scenarios. It should be stressed that the offloading of flights described in this paper is not a tactical process, but rather a shift in airline business strategy. "Offloading" is simply used to describe the process of using demand generation software to shift demand from hub

\footnotetext{
${ }^{*}$ Aerospace Engineer, Systems Modeling and Optimization Branch, Mail Stop 210-15, AIAA Member.
} 
airports to regional airports. This shifting process has been previously referred to in Refs. 6 and 7 as the Point-toPoint-A concept, or PTP-A.

The remaining sections of this paper are organized as follows. Section II details the methodology used, including a description of how the future demand set is generated and what input parameters are applied to the simulation. Section III covers the results of the demand generation and simulation processes. Section IV discusses the conclusions drawn from the results as well as remaining issues.

\section{Methodology}

The methodology used for this study is divided into three sections. Section A describes the demand generation process. The simulation setup and methods are covered in section B. Section C discusses the performance measures used to evaluate the outputs from demand generation and simulation.

\section{A. Demand Generation}

May 17, 2002, a relatively high traffic and low weather impact day, was used as the baseline for current day operations. This day was chosen because several previous studies have used it (including Ref. 6), so its performance in simulation is very well understood. Also, traffic levels on that day are similar to those experienced in today's system. Sensis Corporation's AvDemand software was used to grow future demand from this baseline and distribute flights to regional airports. ${ }^{7}$

The future demand growth rates were taken from the FAA's 2004 Terminal Area Forecast (TAF). The TAF projects growth rates at individual airports throughout the air transportation system for each user class through the year 2015, resulting in a total growth rate of 1.3 times today's traffic levels. ${ }^{8}$ Two different user classes were grown at different rates, representing commercial and general aviation flights. All growth rates were then extrapolated to obtain an overall growth of 1.5 times current levels.

51 hub airports with excessive predicted demand from the TAF were identified that could potentially benefit from offloading flights to secondary airports. Eleven metropolitan areas contained two or more hub airports that were allowed to offload flights to each other. Table 1 shows the number of usable regional airports around the major metropolitan areas in the US. Three main criteria were used to determine whether a regional airport could accept increased demand. The airport needed to be within $50 \mathrm{nmi}$ of the hub airport, it needed to be public use, and it needed to have an asphalt runway in fair or better condition. In addition, flights could only be shifted to airports with runways longer than the minimum takeoff field length for the aircraft type being moved. Airport candidates for offloading were prioritized based on the facility level of that airport, but runway length is a hard constraint, so in some cases regional airports with high facility levels and short runways were unusable for offloading.

The demand generation software begins the offloading process by dividing the day into fifteenminute time bins and identifying those that are over capacity. It then begins shifting flights to regional airports until the demand in each time bin is lowered to match capacity. The algorithm will first attempt to shift

Table 1. Metropolitan areas and associated airports

\begin{tabular}{|l|c|c|}
\hline \multicolumn{1}{|c|}{ Metro Area } & Major Airport(s) & $\begin{array}{c}\text { Number of Regional } \\
\text { Airports }\end{array}$ \\
\hline Atlanta & ATL, PDK & 26 \\
Boston & BOS, MHT & 38 \\
Charlotte & CLT & 22 \\
Chicago & ORD, MDW & 30 \\
Cincinnati & CVG & 22 \\
Cleveland & CLE & 29 \\
Dallas & DFW, DAL & 33 \\
Denver & DEN & 8 \\
Detroit & DTW & 27 \\
Houston & IAH, HOU & 26 \\
Las Vegas & LAS & 9 \\
Los Angeles & LAX, SNA, BUR & 32 \\
Memphis & MEM & 19 \\
Miami & MIA, FLL & 11 \\
Minneapolis-St. Paul & MSP & 21 \\
New York & JFK, EWR, LGA, HPN & 43 \\
Orlando & MCO & 20 \\
Philadelphia & PHL & 43 \\
Phoenix & PHX & 14 \\
Pittsburgh & PIT & 26 \\
Portland & PDX & 17 \\
Saint Louis & STL & 15 \\
Salt Lake City & SLC & 9 \\
San Diego & SAN & 8 \\
San Francisco & SFO, OAK, SJC & 28 \\
Seattle & SEA, BFI & 21 \\
Tampa & TPA & 16 \\
Washington DC & IAD, BWI, DCA \\
\hline
\end{tabular}
flights on the most heavily traveled routes and then will move to flights on less heavily traveled routes. In some cases, there is no available regional airport with enough runway distance and available timeslots to decongest the hub airports. In these cases, the software attempts to split flights onto smaller aircraft so that airports with shorter runways can be utilized. One of the rules applied to the offloading process is that an airport will not accept flights into an already congested time slot. This rule, in addition to runway constraints, makes it impossible to shift certain flights. As a result, after all candidate flights were shifted, there still remained some congested timeslots at several of the hub airports. For a more detailed description of the offloading algorithm, see Ref. 7. 


\section{B. Simulation}

The output of the demand generation process is a set of flights that are used as input to the simulation software. NASA's Airspace Concept Evaluation System (ACES) v4.6.2 was used to carry out the simulation. ${ }^{9}$ ACES allows high fidelity simulation of all of the itinerant flights in the national air transportation system for an entire day. Physics-based models are used to simulate the en-route portion of flights, while queuing models simulate flights in the terminal area. Airport arrival and departure capacities, as well as sector capacities can be set to represent current or future configurations of the system. For a thorough description of the ACES software, see Ref. 9.

For this experiment, it was assumed that an automated conflict detection and resolution system would be in place in the future, allowing for higher sector capacities. Previous studies indicate that up to a 3 times increase in sector capacity is possible with such a system. ${ }^{10}$ A 2 times increase was used for this study as a conservative estimate of what the future system will allow.

The only improvements assumed at the airports were those planned in the FAA's 2004 Operational Evolution Plan (OEP). These improvements include new runways, equipment, technologies, and procedures planned for the 34 busiest airports in the continental United States. In ACES, they were represented as up to a 61 percent increase in airport capacity, depending on the improvements planned at each airport. The capacity increases over current day are shown in Fig. 1.

Two simulations with and without offloading were run at future demand levels for comparison. The first assumed the increases in airport and sector capacity, but allowed unconstrained traffic growth without offloading flights from busy hub airports. The second kept the increased capacities and added the option of shifting flights during congested timeslots to other airports.

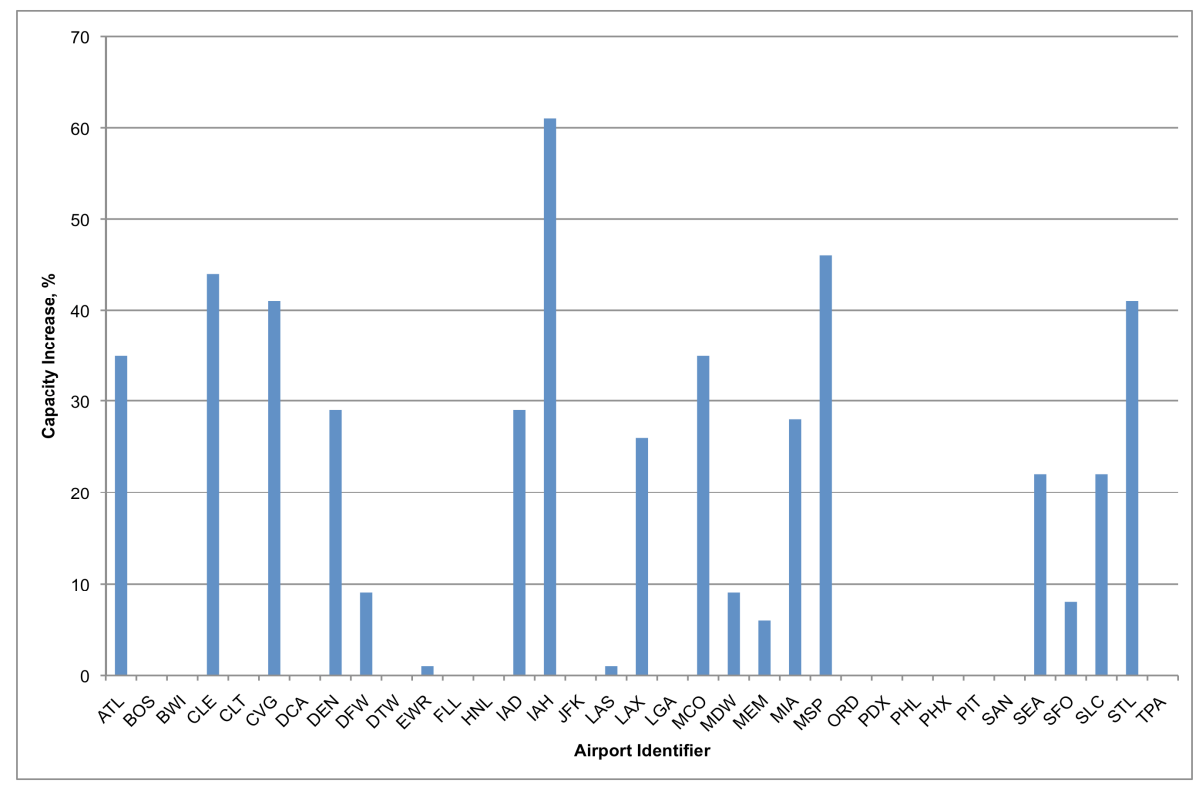

Figure 1. Predicted capacity increases at the OEP 34 airports $^{2}$

\section{Performance Measures}

The performance of the system under both future demand scenarios was evaluated using predictive metrics and actual simulation outputs. The predictive metrics give an idea of where congestion-induced delays are likely to occur without actually running the simulation, while the simulation outputs provide the actual simulated delay.

The results of these two simulations were compared against each other and against a simulation of current day operations. Particular attention was paid to the Washington, DC metro area. Focus was placed there for two reasons. First, there are three major airports in this region, which can all offload flights to each other. Second, there are several secondary airports in the region that can accept offloaded flights from Baltimore, Dulles, or Reagan National airports.

Three predictive metrics were calculated prior to running the simulation. The first predictive metric was minimum required capacity, which is a measure of how much arrival or departure capacity is needed at an airport, given a traffic demand set and a maximum allowable delay for individual flights as well as for the airport overall. ${ }^{11}$ For this study the maximum allowable delays were set at 10 minutes for a flight and 3 minutes average for an airport. This metric has also been used to determine the minimum required capacity for sectors; however, it was only applied to airports for this study.

The second predictive metric was the queuing delay. This metric predicts daily arrival or departure delay based on how the instantaneous demand at an airport compares to its available capacity. When demand exceeds capacity in a 15-minute time bin, a queue builds as aircraft are delayed at the airport. The number of aircraft in the airport's queue is multiplied by the size of the time bin to obtain the total minutes of delay accrued for each time bin. During 
times when the demand is less than capacity, the queue size decreases, but cannot go below zero. The delay accrued for all time bins at an airport is then summed and divided by the number of arrivals or departures at that airport to obtain the daily per-flight queuing delay.

The third predictive metric is the 16-hour demand to capacity ratio. This metric is a measure of how much of an airport's daily capacity is used. The demand for a whole day is compared against the effective daily capacity, which is defined as the amount of traffic that can be handled during 16 hours with the airport operating at maximum capacity. Ref. 6 has shown that as this ratio approaches 70 percent, delays begin to increase dramatically.

Delay was used as the primary indicator of system performance for the simulation results. In ACES, aircraft delay is defined as the difference between actual arrival time and unconstrained arrival time. This is slightly different from the FAA's definition of delay, which is the difference between actual and scheduled arrival time. In addition to delay, percent capacity used and percent on-time performance were calculated from the simulation outputs. These are metrics that the FAA tracks to evaluate an airport's performance. ${ }^{12}$

Percent capacity used is an efficiency metric used to measure the portion of an airport's capacity that is being used at any given hour of the day. Percent on-time performance is a customer satisfaction metric used to measure the number of flights that arrive on time at a given airport. An on-time arrival is defined by the FAA's Aviation System Performance Metrics as one that arrives at the gate no more than fifteen minutes after its scheduled arrival time.

\section{Results}

The results are presented in three sections. Section A details the results of the demand generation process. Section B discusses metrics that were calculated to predict the performance of the system based on the results of demand generation only. The results of the simulations are presented in section $\mathrm{C}$.

\section{A. Demand Generation}

All hub airports where the offloading algorithm was applied were able to reduce their traffic levels. In general, the congested hub airports were able to reduce demand to match capacity. Table 2 shows how flights were offloaded from the three major Washington DC area airports-Washington Dulles (IAD), Baltimore International (BWI), and Reagan National (DCA). Although 36 regional airports are available in the Washington DC area, only three were used by the offloading algorithm: Manassas Regional (HEF), Eastern West Virginia Regional (MRB), and Martin State (MTN). This mainly occurred because the additional 33 regional airports did not have long enough runways to accommodate the flights that needed to be shifted, or because HEF, MRB, and MTN had higher priorities as receiving airports.

Table 2. Flights shifted at Washington DC area airports, divided into departures/arrivals.

\begin{tabular}{|c|c|c|c|c|c|c|c|c|c|}
\hline & \multicolumn{6}{|c|}{ Offloaded To } & \multirow[b]{2}{*}{ Split Total From } & \multirow[b]{2}{*}{ Total From } \\
\hline & & IAD & BWI & DCA & HEF & MRB & MTN & & \\
\hline \multirow{6}{*}{$\begin{array}{l}\frac{0}{0} \\
\frac{0}{0} \\
\frac{\pi}{0} \\
\frac{0}{4} \\
\frac{4}{0}\end{array}$} & IAD & & $3 / 4$ & $0 / 0$ & $31 / 31$ & $7 / 10$ & $213 / 212$ & $254 / 257$ & 511 \\
\hline & BWI & $6 / 2$ & & $6 / 3$ & $4 / 4$ & $33 / 30$ & $58 / 68$ & $107 / 107$ & 214 \\
\hline & DCA & $1 / 0$ & $5 / 4$ & & $1 / 1$ & $0 / 1$ & $1 / 0$ & $8 / 6$ & 14 \\
\hline & HEF & $0 / 0$ & $0 / 0$ & $0 / 0$ & & $0 / 0$ & $0 / 0$ & $0 / 0$ & 0 \\
\hline & MRB & $0 / 0$ & $0 / 0$ & $0 / 0$ & $0 / 0$ & & $0 / 0$ & $0 / 0$ & 0 \\
\hline & MTN & $0 / 0$ & $0 / 0$ & $0 / 0$ & $0 / 0$ & $0 / 0$ & & $0 / 0$ & 0 \\
\hline & Split Total To & $7 / 2$ & $8 / 8$ & $6 / 3$ & $36 / 36$ & $40 / 41$ & $272 / 280$ & & \\
\hline & Total To & 9 & 16 & 9 & 72 & 81 & 552 & & \\
\hline
\end{tabular}

Table 2 is organized such that flights from the airport on the left are offloaded to the airports along the top. The first number in each box is the number of departing flights that are offloaded, while the second is the number of arriving flights offloaded. For example, IAD offloads 213 departures and 212 arrivals to MTN. The grey boxes on the right and on the bottom are the total number of flights offloaded. From IAD, for example, a total of 254 departures and 257 arrival flights are shifted for a total of 511 flights. As the table shows, IAD offloads the most flights from the DC area airports. Of the three major airports in the area, it was the most overloaded after the traffic was grown to future levels. Since MTN has the longest runway of the regional airports in the area and is thus more capable of accepting flights on larger aircraft, it accepts the most flights.

Although the offloading process reduced demand levels at the large hubs, several airports still had demand that exceeded capacity. As expected, airports such as Las Vegas-McCarran, which do not have many usable regional airports surrounding them, remained the most over-capacity."

* This study did not consider the Ivanpah Valley Airport, which is planned in southern Nevada to relieve traffic from Las Vegas-McCarran Airport 


\section{B. Predictive Metrics}

The three predictive metrics previously discussed-minimum required capacity, queuing delay, and 16-hr demand-to-capacity ratio - were calculated before running the simulation. All three metrics were calculated for each of the 34 mainland OEP airports using the same set of flights as that used as input for the simulation.

In Fig. 2, the first bar, marked " $1.5 \mathrm{x}$ Unconstrained,"

represents the minimum required capacity for each airport for the case where traffic is grown to $1.5 \mathrm{x}$ current levels without utilizing regional airports. The second bar, marked "1.5x Demand Shifted," represents the case where flights have been shifted to regional airports, representing the alternate future demand scenario. The third bar, "OEP Capacities," shows what the predicted capacity for each hub

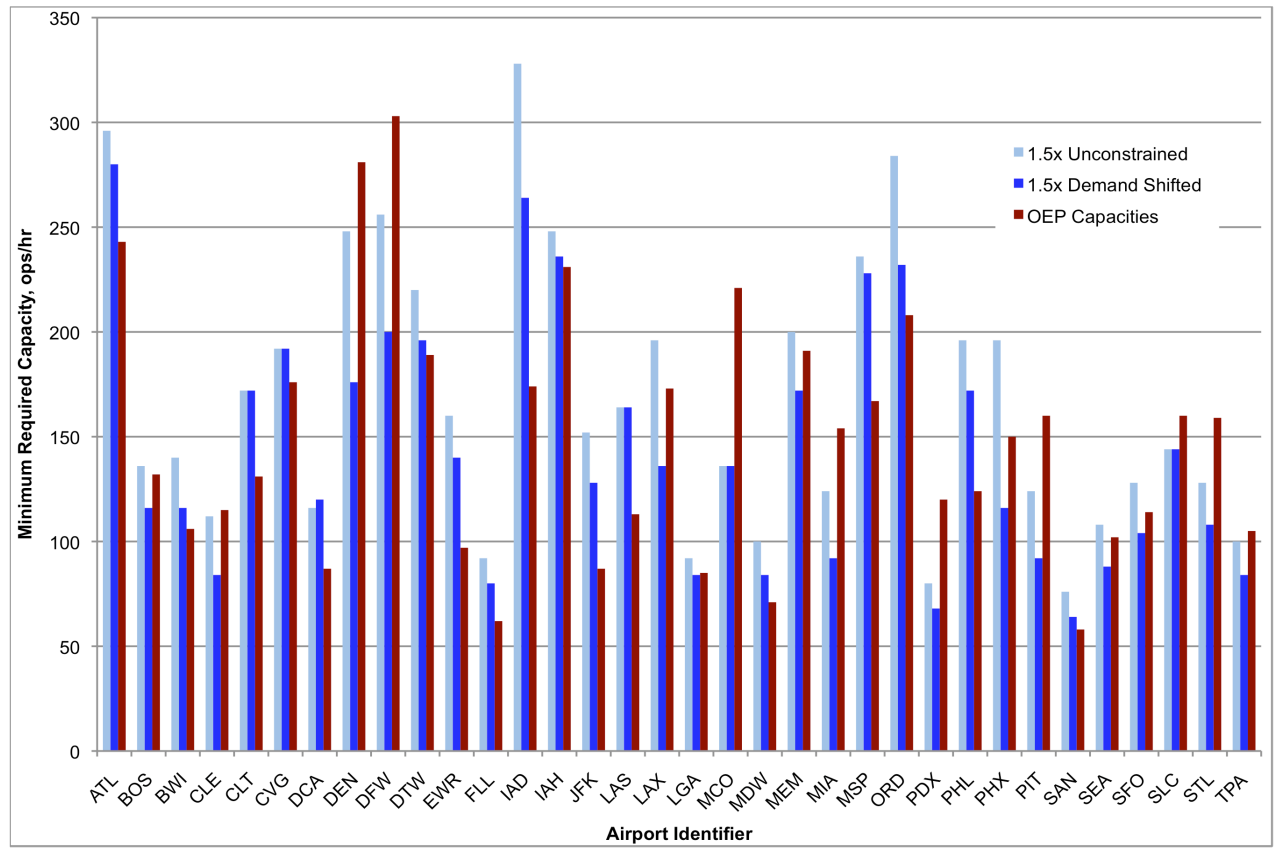

Figure 2. Minimum required capacity at the OEP 34 airports airport will be.

In Figs. 3 and 4, the "1.5x Unconstrained" and "1.5x Demand Shifted" bars represent the given metrics without and with offloading, respectively. In both cases, the predicted OEP capacities were used to calculate the metrics. The "1x Baseline" bar in Figs. 3 and 4 represents the given metric as calculated when the simulation is run at current

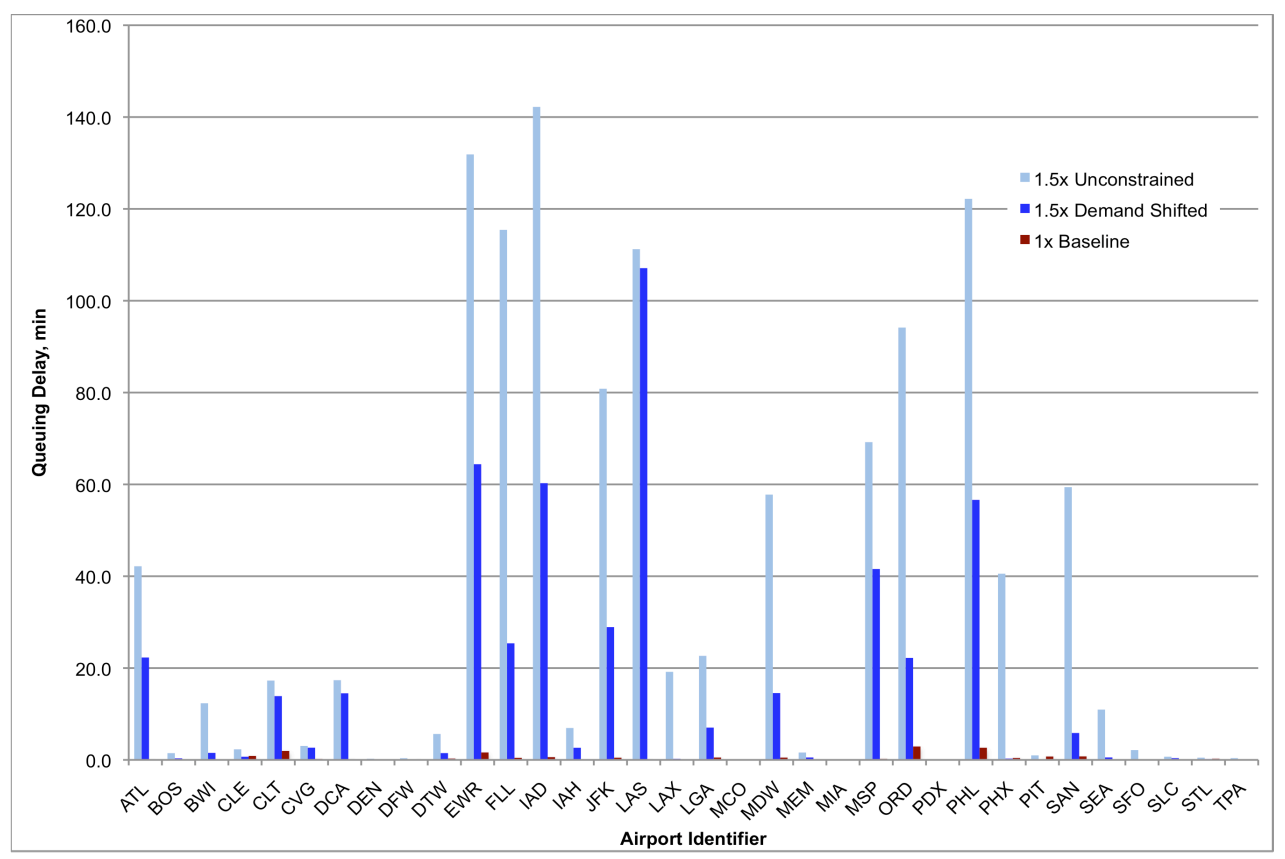

Figure 3. Predicted arrival queuing delay per flight at the OEP 34 airports demand levels and current airport capacities. Ref. 13 has shown that the simulation of the baseline day will provide a good basis of comparison for the performance of the system in the future demand scenario.

It is clear that at most of the airports listed, the extended use of regional airports leads to a reduction in minimum required capacity, shorter queuing delays, and smaller 16-hour demand-to-capacity ratios. Of the 34 
OEP airports, 28 see a decrease in minimum required capacity with reductions of up to 41 percent in this metric. In several cases, such as at BOS, PHX, and LAX, the minimum required capacity is brought below the predicted OEP capacity, indicating delays should be below their target values at those airports. Large queuing delays are significantly reduced at most of the airports where they occur in the unconstrained growth case. Figure 3 shows that

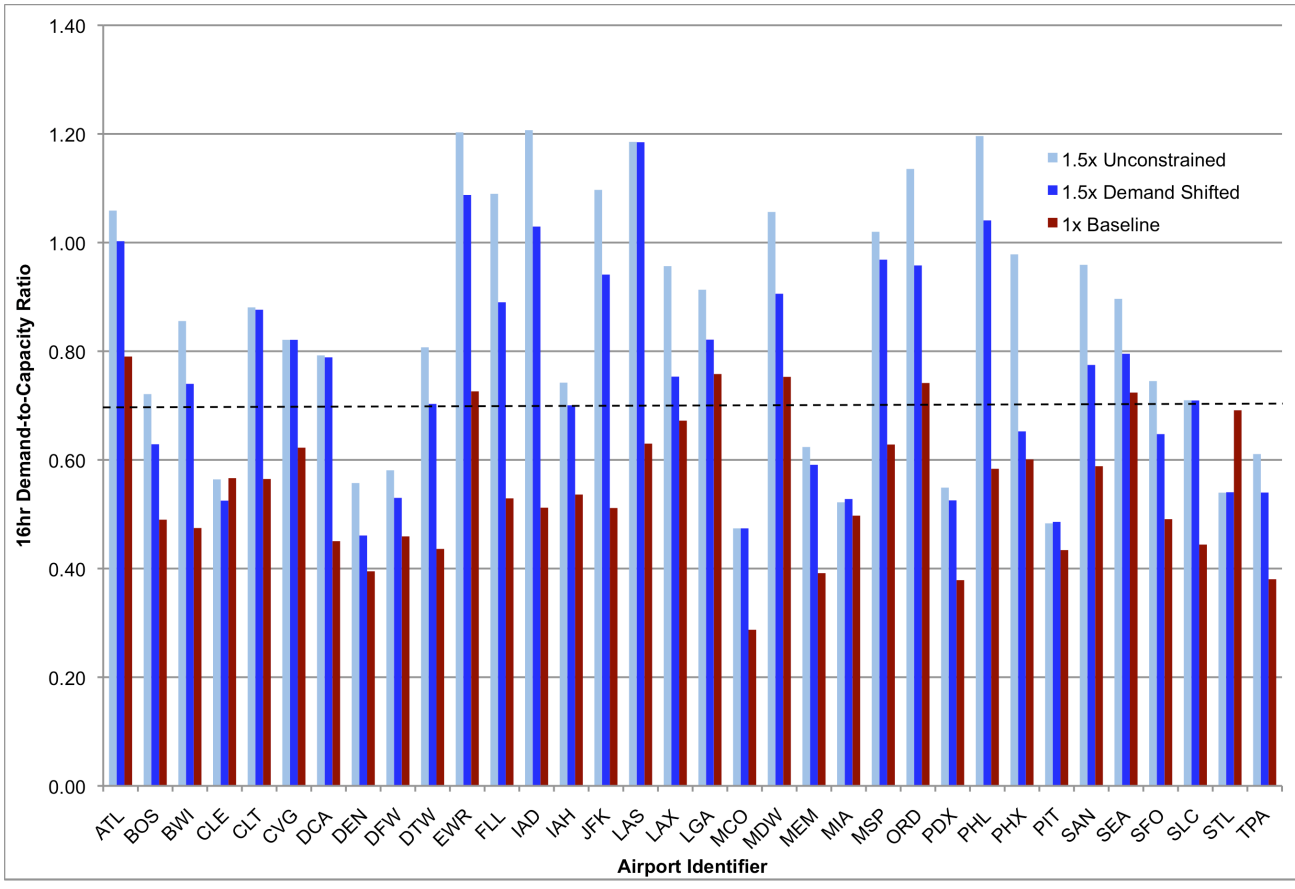

Figure 4. 16-hour demand-to-capacity ratio at the OEP 34 airports the queuing delay calculated for any airport in the $1 \mathrm{x}$ baseline case is approximately 3 minutes. In a few cases - notably BWI, LAX, and PHXsignificant queuing delays are brought below this value once flights are offloaded. As Fig. 4 shows, many OEP airports have 16hour demand-tocapacity ratios well above 70 percent in the unconstrained growth case, and some even exceed this threshold in the baseline $1 \mathrm{x}$ case. All hubs that exceed a 70 percent demand-

to-capacity ratio in the unconstrained growth case see a reduction in this metric once flights are offloaded, and several have this ratio drop to near or below 70 percent. A dotted line is drawn on Fig. 4 to show this 70 percent threshold.

Several problem airports still remain; the most notable of these are Las Vegas and those in the New York and Washington DC areas. At these airports, there are large mismatches between the minimum required capacities and the predicted OEP capacity, as well as large queuing delays and demand-to-capacity ratios well above $70 \%$. These airports can be expected to exhibit large delays in simulation. Also, delays at these airports can be expected to cascade throughout the system, causing a significant increase in delays at other airports. None of the predictive metrics accounts for networking effects between airports, so delays from this cascading effect do not appear before running the simulation.

\section{Simulation}

Simulation of 1x traffic on the baseline day, May 17, 2002, results in an average delay of 5 minutes per flight. In Ref. 6, a mean delay of 10 minutes per flight has been used as the acceptable maximum. When air traffic levels are grown 50 percent above today's levels without utilizing regional airports, the simulation shows that system-wide delays are completely unacceptable. In this scenario, the average per-flight delay is 44 minutes across the system. Once secondary airports are used to offload flights, the average per-flight delay drops to 18 minutes - still unacceptable, but a significant improvement over the unconstrained growth case.

Arrival delays were computed for all 34 OEP airports and are shown in Fig. 5. The plot shows that in the 1x baseline case, no airport has an average per-flight arrival delay greater than 20 minutes. In the $1.5 \mathrm{x}$ unconstrained growth case, arrival delays at many airports are very large. In the real system, delays of this length would correspond to many canceled flights, but the simulation is only able to impose large delays, not cancellations. Once demand is shifted in the $1.5 \mathrm{x}$ case, all of the extreme delays are reduced. In some cases-FLL, PHX, and SANthese delays are reduced to acceptable levels, while in other cases, such as EWR, IAD, and LAS, delays remain very large. From the results shown in Fig. 5, it is clear that certain airports can alleviate their congestion problems by offloading excess demand growth to regional airports, while others cannot. These results are consistent with the 
queuing delay predictions shown in Fig. 3., as large delays appear where they were predicted to occur. At airports where flight offloading eliminated large queuing delays, simulated delays were also greatly reduced.

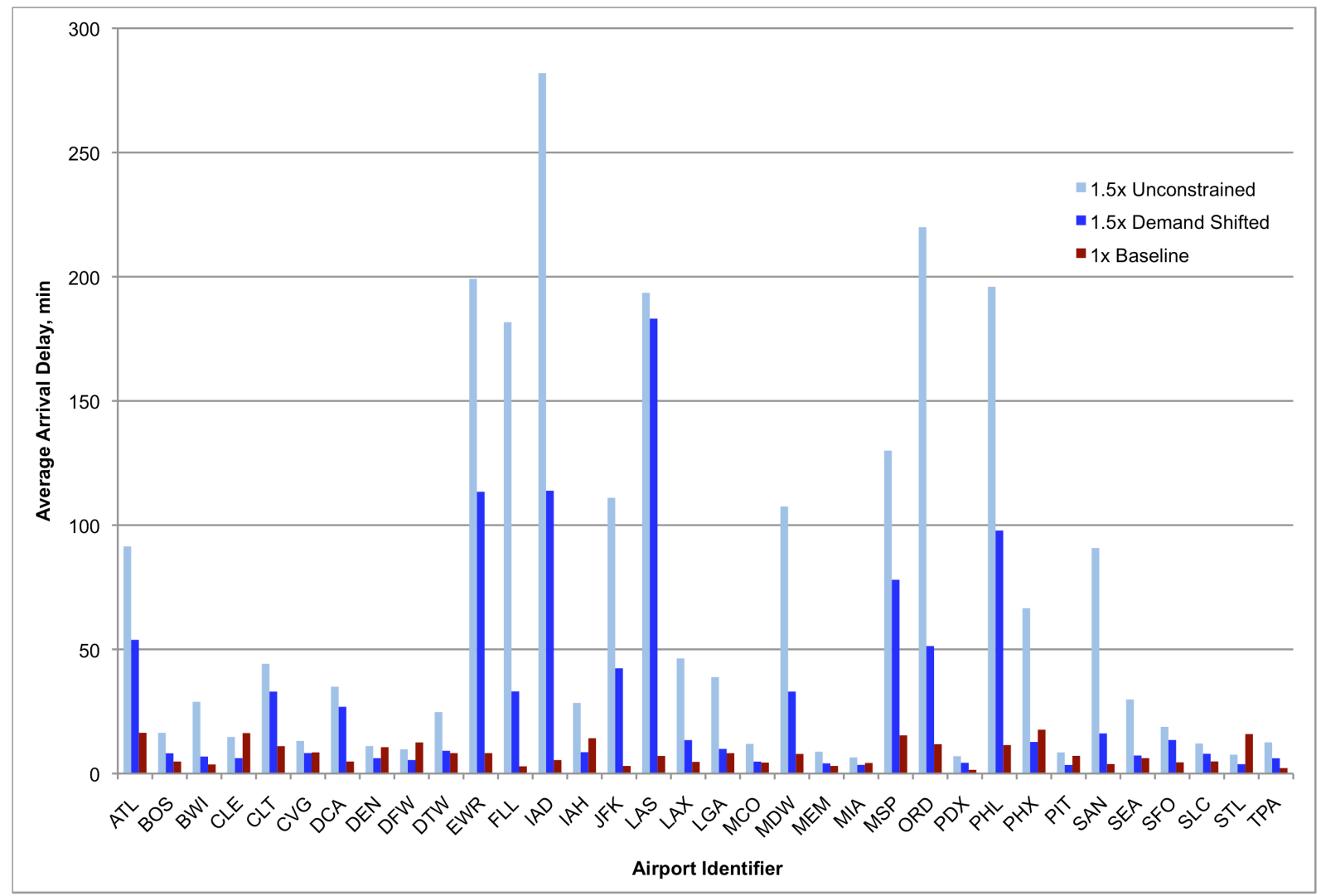

Figure 5. Simulated per-flight arrival delay at the OEP 34 airports

Again, the Washington DC area airports were a point of focus. Figure 6a shows the scheduled and actual demand at Washington Dulles airport without shifting flights to secondary airports. The large number of actual operations towards the end of the simulation above the scheduled operations line shows that many flights are delayed. Figure $6 \mathrm{~b}$ shows that the shifting of flights significantly reduces the number of scheduled operations during peak hours. Also, the number of actual gate operations more closely tracks the scheduled gate operations, indicating a reduction in delays.

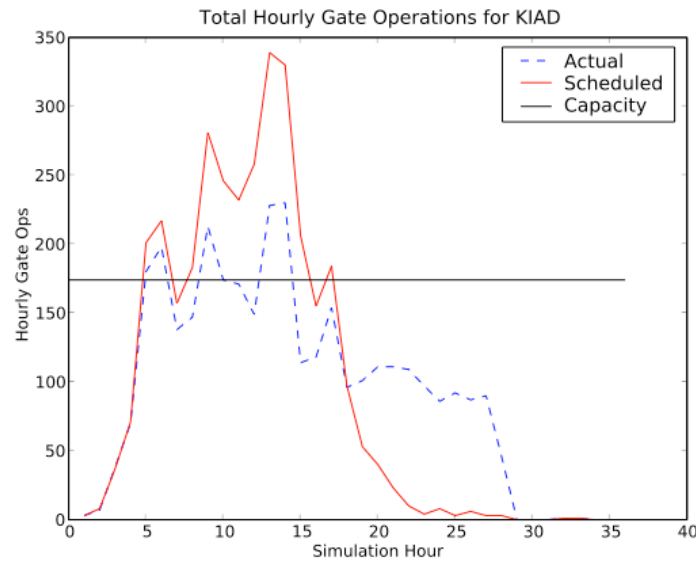

(a)

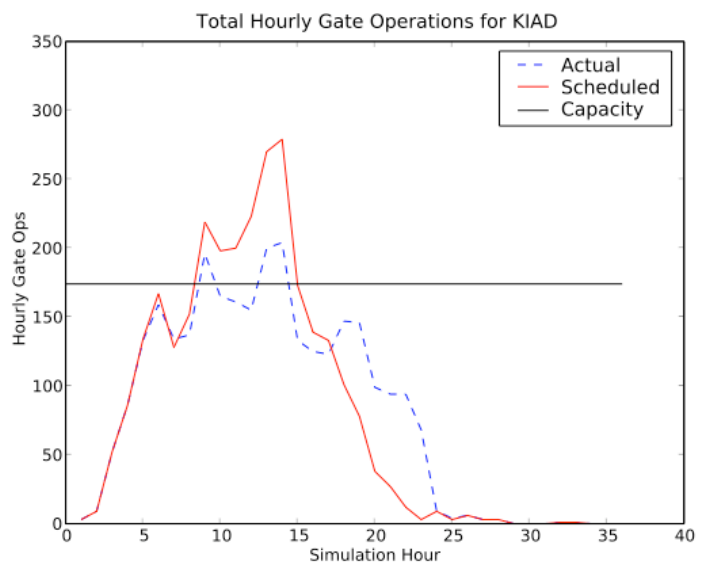

(b)

Figures 6a, b. Demand at Washington Dulles International Airport (IAD) before and after offloading 
Figure 7a shows the scheduled and simulated demand at Martin State Airport before flights are shifted. This airport accepts the bulk of the traffic that is offloaded from the major Washington DC area airports, as previously shown in Table 1. As the plot shows, the scheduled demand at this airport prior to offloading is significantly lower than the capacity. The traffic that exists there is largely general aviation. Figure $7 \mathrm{~b}$ shows that after flights are shifted, scheduled demand nearly reaches capacity during peak hours. Actual gate operations almost exactly track scheduled operations, indicating that no significant delay is accrued as a result of the increased scheduled demand at the secondary airport.

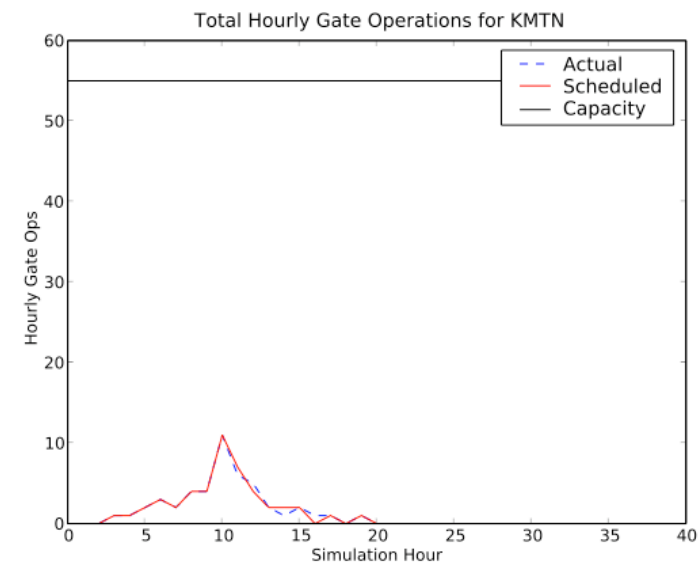

(a)

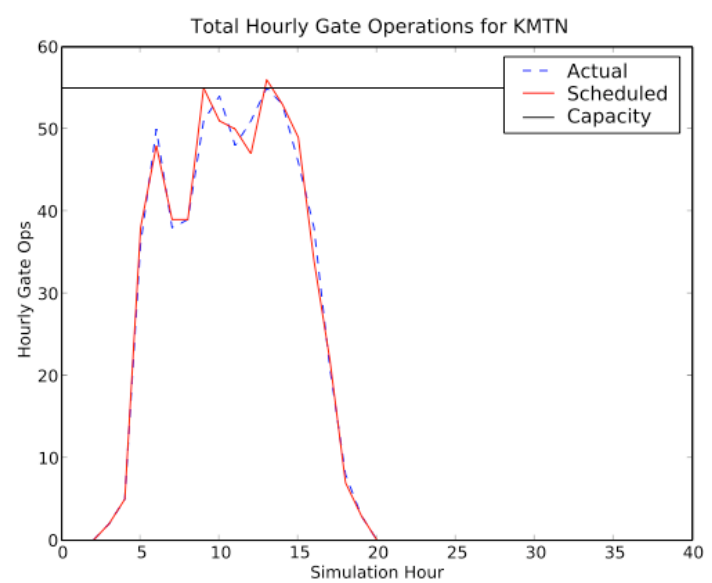

(b)

Figures 7a, b. Demand at Martin State Airport, Baltimore, MD (MTN) before and after offloading

Figure 8 shows the percent capacity used during simulation at Washington-Dulles and Martin State Airports before and after flights were shifted. As the pre-shift plot shows, Washington-Dulles uses nearly one hundred percent of its capacity for over ten hours during the day, while Martin State never exceeds 20 percent of its hourly capacity. The post-shift plot shows that Washington-Dulles remains heavily loaded for a large portion of the day; however, the time during which the airport is running at full capacity is significantly reduced. The demand at Martin State is sharply increased, such that it is running near full capacity for several of the busiest hours of the day.

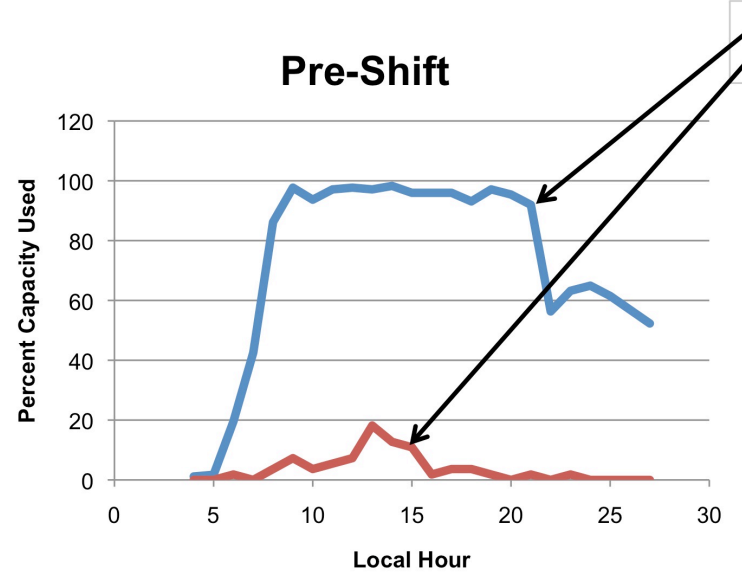

(a)

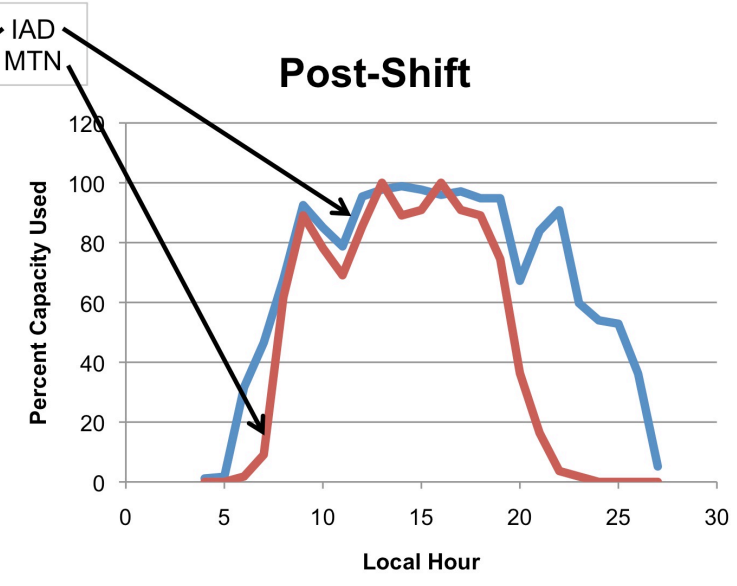

(b)

Figure 8a, b. Hourly percent capacity used at Washington-Dulles (IAD) and Martin State Airport (MTN) before and after offloading

The reduction in traffic around the hub airports can be seen in a visualization of the traffic flows. Figures $9 \mathrm{a}$ and $b$ show color diagrams of the traffic flows for the entire simulation day as flown in the ACES software. Focus is on the Washington DC and New York metro areas. Warmer colors indicate higher traffic counts. Not represented here is the added complexity in the terminal area due to increased traffic at the regional airports. 


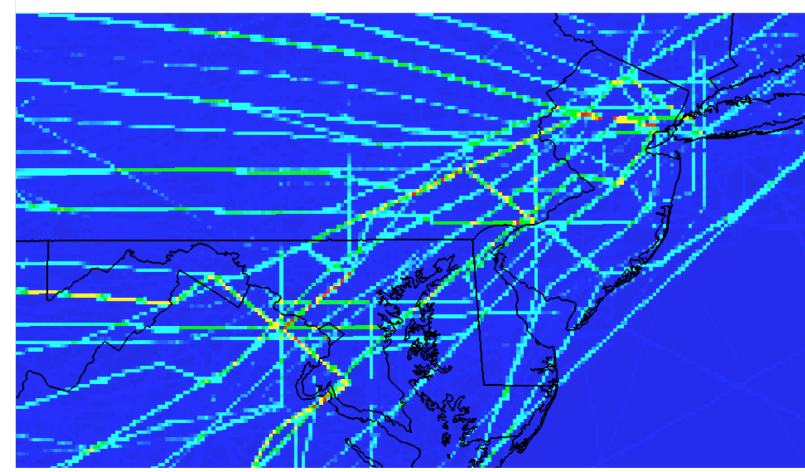

(a)

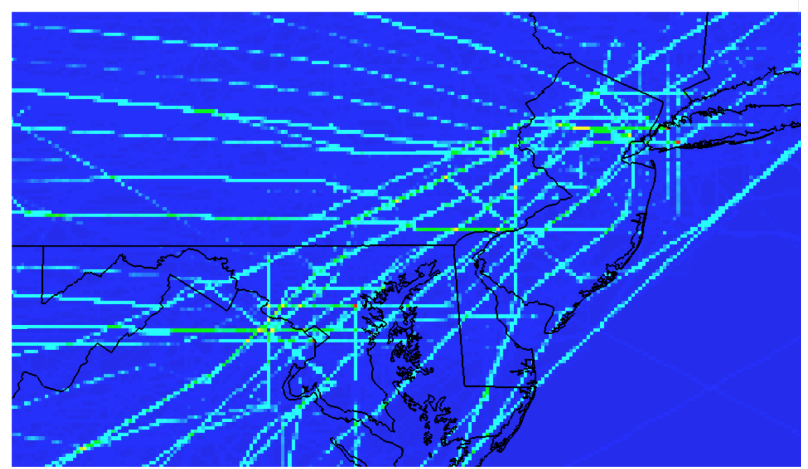

(b)

Figures 9a, b. Northeast corridor air traffic loading before and after offloading

The data represented in Figs. 9a and $\mathrm{b}$ show that traffic is heavier coming out of the major airports when no flights are offloaded to regional airports. If traffic utilizes the regional airports more, the congestion around the metro airports decreases. Another feature to note is the uniformity of the flight tracks. Since there is currently no uncertainty model built into the ACES simulation software, every flight between a given set of waypoints will fly almost the exact same trajectory. This is not exactly the way aircraft fly in the actual system, but real flight track data would look quite similar.

The FAA tracks several "customer satisfaction" metrics to gauge how well the air transportation system is performing from a passenger standpoint. These metrics include, among others, percent on-time arrivals, en route delays, and airport efficiency. The on-time performance as simulated for the OEP 34 airports is plotted in Fig. 10.

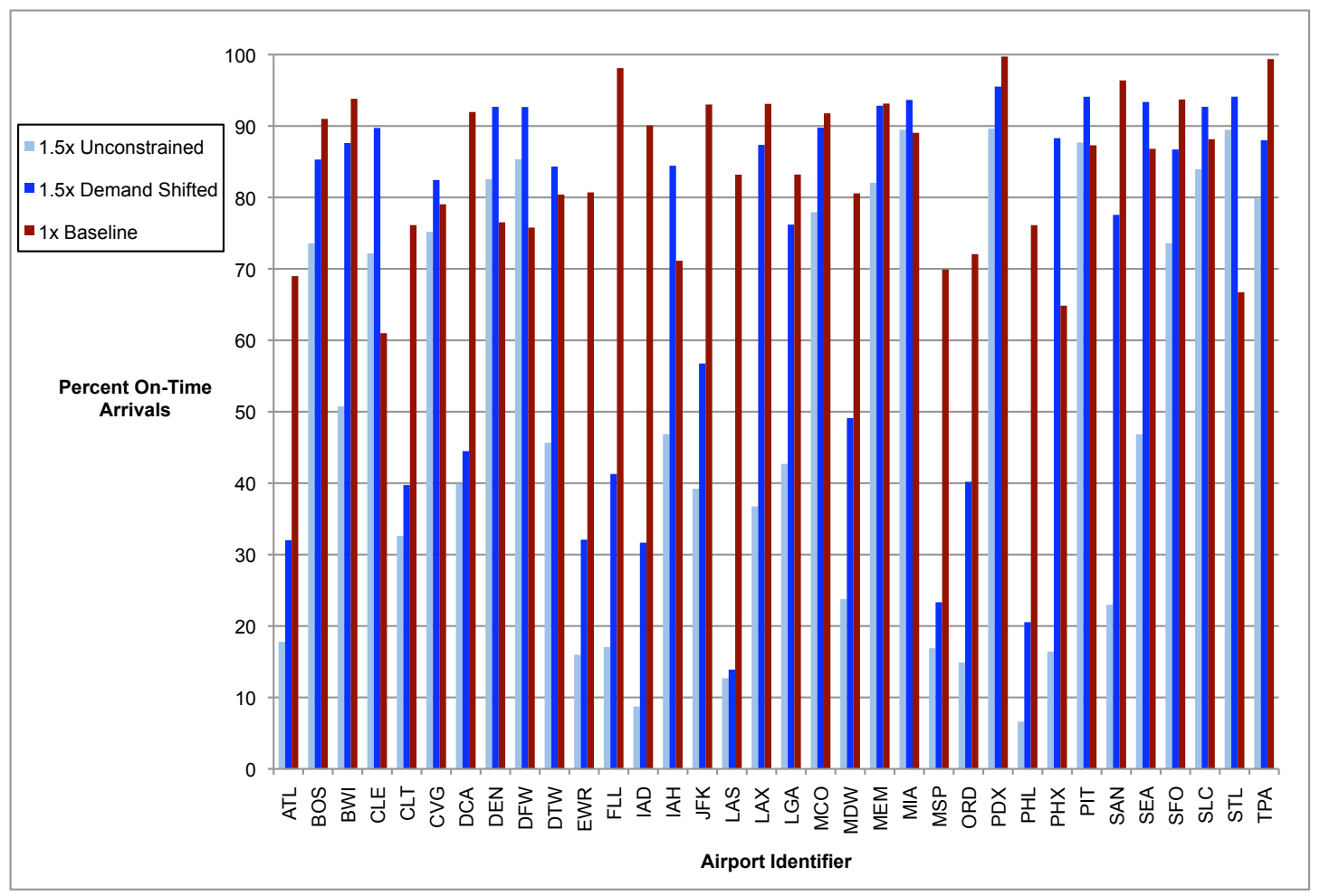

Figure 10. On-time arrival performance at the OEP 34 airports

The light blue columns indicate on-time performance at the hub airports for the unconstrained $1.5 \mathrm{x}$ future demand scenario. The dark blue columns represent the hub airports' performance after demand growth is shifted to regional airports. The red columns represent airports' on-time performance for a baseline run with traffic levels and airport capacities as they were observed on the demand day. As Fig. 10 shows, most of the 34 airports show 
significant improvements in on-time performance after demand is offloaded. As could easily be expected, the airports that show the least improvement in on-time arrival performance are those that are least able to shift demand to regional airports.

Overall, the simulation results show significant improvements in the performance of the system if excess demand is offloaded to regional airports. Delays are considerably reduced at almost all major airports, resulting in much improved on-time performance over the case where demand is allowed to grow unconstrained. In addition, the simulations show that the regional airports that accept offloaded flights from congested hubs do so without experiencing increases in delay.

\section{Conclusions}

Airlines are beginning to utilize regional airports to grow air traffic demand in metropolitan areas, rather than adding operations at already congested hub airports. To examine the effects of this change in business strategy, a study was carried out to simulate a large-scale future demand shift to regional airports. Demand levels were grown by fifty percent from May 17, 2002, and two possible demand scenarios were generated. The first was created by growing traffic unconstrained based on FAA predictions. To represent a possible alternative future, a large portion of traffic was then shifted to regional airports to generate the second demand scenario. The number of flights available for shifting varied by hub airport, depending on the availability of surrounding regional airports.

Metrics were calculated to predict the performance of the system before simulations were run. These metrics showed that once flights were offloaded from the busy hub airports, delays at many of the nation's major airports could be expected to be much less severe than for the case where air traffic demand is grown unconstrained. In general, these metrics predicted that problems would remain at locations where the flight-offloading algorithm was unable to significantly reduce demand at the hub airport. The predictions of these metrics were then confirmed by the simulation data.

Simulations of the two possible future demand scenarios were carried out using NASA's ACES software. Metrics were calculated to evaluate the performance of the system when loaded with the two different demand sets. The simulation results show an average system-wide per-flight delay of 44 minutes with unconstrained demand at $1.5 x$ today's levels. After demand is shifted to regional airports, the per-flight delay drops to 18 minutes. Although this level of delay exceeds the acceptable maximum of 10 minutes, it represents a large improvement over the unconstrained growth case. Airport-level metrics, including delays, percent on-time performance and percent capacity used, were also calculated for the two demand scenarios and compared against the performance of the present-day system. When flights are offloaded, these metrics show considerable improvement over the unconstrained growth case.

While several airports remain over capacity and would not be able to operate under the conditions tested, average delays across the system are not extreme. This result suggests that while offloading to regional airports is not a single solution to accommodating future air traffic demand levels, it can have a very significant benefit.

\section{Acknowledgments}

The author would like to thank Mr. Michael Downs for his assistance in setting up and running the air traffic simulations, Dr. Alex Huang for his technical support of the AvDemand software, and Dr. Gano Chatterji for his development of the queuing delay metric.

\section{References}

\footnotetext{
${ }^{1}$ Federal Aviation Administration, “Terminal Area Forecast Summary, Fiscal Years 2007-2025,” FAA HQ-08371, 2008.

${ }^{2}$ Federal Aviation Administration, "Airport Capacity Benchmark Report 2004," Federal Aviation Administration, Washington DC, 2004.

${ }^{3}$ McCartney, Scott, "Sending Fliers Up the River to Ease Traffic," The Wall Street Journal, March 4, 2008, p. D1.

${ }^{4}$ Bonnefoy, Philippe and R. John Hansman, Jr., "Scalability and Evolutionary Dynamics of Air Transportation Networks in the United States," AIAA Aviation Technology, Integration and Operations Conference, AIAA, Washington DC, 2007.

${ }^{5}$ Mozdzanowska, Aleksandra and R. John Hansman, "Growth and Operating Patterns of Regional Jets in the United States," Journal of Aircraft, Vol. 42, No. 4, Jul-Aug 2005, pp. 858-864.

${ }^{6}$ VAMS Project Office, "Virtual Airspace Modeling and Simulation System-Wide Concept Report," NASA Ames Research Center, Moffett Field, CA, 2006.

${ }^{7}$ Huang, Alex and David Schleicher, "A Flexible Demand Generation Application for NextGen Concept Evaluation," AIAA modeling and Simulation Technologies Conference and Exhibit, AIAA, Washington DC, 2007.
} 
${ }^{8}$ Federal Aviation Administration, "FAA Aerospace Forecasts Fiscal Years 2005-2016, Executive Summary," Federal Aviation Administration, Washington DC, 2005.

${ }^{9}$ Meyn, Larry, et al., "Build 4 of the Airspace Concept Evaluation System," AIAA Modeling and Simulation Technologies Conference and Exhibit, AIAA, Washington DC, 2006.

${ }^{10}$ Farley, Todd and Heinz Erzberger, "Fast-Time Simulation Evaluation of a Conflict Resolution Algorithm under High Air Traffic Demand," USA/Europe Air Traffic Management R\&D Seminar, Eurocontrol, Brussels, Belgium, 2007.

${ }^{11}$ Zelinski, Shannon and Tom Romer, "Estimating Capacity Requirements for Air Transportation System Design," USA/Europe Air Traffic Management R\&D Seminar, Eurocontrol, Brussels, Belgium, 2007.

${ }^{12}$ Federal Aviation Administration, “Aviation System Performance Metris, (ASPM)," http://www.aspm.faa.gov/, May 2009.

${ }^{13}$ Zelinski, Shannon and Larry Meyn, "Validating the Airspace Concept Evaluation System for Different Weather Days," AIAA Modeling and Simulation Technologies Conference and Exhibit, AIAA, Washington DC, 2006. 